Hardy Ramanujan Journal

Vol. 28(2005) 10-29

\title{
ON AN EXPONENTIAL SUM INVOLVING THE MÖBIUS FUNCTION
}

\author{
H. MAIER(1) AND A. SANKARANARAYANAN ${ }^{(2)}$
}

\begin{abstract}
In this paper we study the upper bound for the absolute value of the exponential sum related to the Möbius function unconditionally and present some interesting applications also.
\end{abstract}

12

\section{Introduction}

Let $\mathbf{e}(\alpha)$ denote $e^{2 \pi i \alpha}$. We consider the exponential sum

$$
S(x, \theta)=\sum_{n \leq x} \mu(n) \mathbf{e}(n \theta) .
$$

R.C. Baker and G. Harman (see [1]) studied this sum under the hypothesis that for every Dirichlet character $\chi, L(s, \chi)$ has no zeros in the half-plane $\sigma>a$. They proved THEOREM A. Suppose that the above hypothesis holds for every Dirichlet character $\chi$. Then

with

$$
\max _{\theta}|S(x, \theta)| \ll x^{b+\epsilon}
$$

$$
b=\left\{\begin{array}{lll}
a+\frac{1}{4} & \text { if } & \frac{1}{2} \leq a<\frac{11}{20}, \\
\frac{4}{5} & \text { if } & \frac{11}{20} \leq a<\frac{3}{5}, \\
\frac{1}{2}(a+1) & \text { if } & \frac{3}{5} \leq a<1 .
\end{array}\right.
$$

For other interesting related results we refer to [2], [4], [11] and [13]. Throughout the paper, the integer $d$ is a divisor of $q, \chi^{*}$ denotes a Dirichlet character modulo $q$, $\chi_{d}$ denotes the principal character modulo $d$ and $\chi$ denotes a character modulo $\left(\frac{q}{d}\right)$. The constant $A$ is a generic absolute constant which need not be the same at each occurrence. $\epsilon$ and $\delta$ denote arbitrarily small positive constants. We use $\|\theta\|$ to mean that

\footnotetext{
${ }^{1}$ AMS Subject Classification : primary $11 \mathrm{M}$, secondary 11 M06, 11 N05.

${ }^{2}$ Key words : Dirichlet $L$-function, Möbius function, Exponential sum, Zero-free region.
} 


$$
\|\theta\|=\min _{m \in \mathbb{Z}}|\theta-m| .
$$

Throughout the paper, we assume that $x \geq x_{0}$ (where $x_{0}$ is sufficiently large) and the modulus $q \geq q_{0}(\geq 10)$.

Now, the main goal of this paper is to give an unconditional upper bound in terms of zero density functions of Dirichlet L-functions for $|S(x, \theta)|$. Let

$$
N(\alpha, T, \chi)=\#\{\rho=\beta+i \gamma: L(\rho, \chi)=0, \beta \geq \alpha>0,|\gamma| \leq T\} .
$$

We prove

THEOREM 1. Let $q$ be a positive integer and $\theta$ be a real number. Then for any rational number $\frac{r}{q}$ with $(r, q)=1$ and for any fixed $\alpha$ satisfying $\frac{1}{2}+\delta \leq \alpha \leq 1-\delta$ (with $\delta$ being any small positive constant), we have

$$
\begin{aligned}
S & =: S(x, \theta) \\
& \ll q^{-\frac{1}{2}} x^{1+10 \epsilon}+\sum_{d \mid q}\left(\frac{q}{d}\right)^{\frac{1}{2}} \cdot \frac{1}{\phi\left(\frac{q}{d}\right)}\left(1+x\left|\theta-\frac{r}{q}\right|\right) \times
\end{aligned}
$$

$$
\times\left(\max _{1 \leq T \leq\left(\frac{x}{d}\right)^{3}} T^{-1}(q T)^{\epsilon} \int_{\frac{1}{2}}^{1}\left(T \phi\left(\frac{q}{d}\right)\left(\frac{x}{d}\right)^{\alpha+\epsilon}+\left(\frac{x}{d}\right)^{\sigma+\epsilon} \sum_{\chi} N\left(\sigma, T, \chi \chi_{d}\right)\right) d \sigma\right) .
$$

Here $\chi_{d}$ denotes the principal character modulo $d$ and the functions $\chi_{d}$ are characters modulo $q$.

To strengthen our Theorem 1 above, we present here a few applications as Theorems 2, 3 and 4 in the following. Note that Theorem 2 is unconditional whereas Theorem 3 depends on the Average Zero-Density Hypothesis. Since they depend on the type of $\theta$, let us recall this notion (see page 121 of [7] for more details). Let $\psi$ be a non-decreasing positive function that is defined at least for all positive integers. The irrational number $\theta$ is said to be of type $<\psi$ if $q\|q \theta\| \geq \frac{1}{\psi(q)}$ holds for all positive integers $q$. If $\psi$ is a constant function, then an irrational $\theta$ of type $<\psi$ is also called of constant type. Let $\eta_{1}$ be a positive real number or infinity. The irrational number $\theta$ is said to be of type $\eta_{1}$ if $\eta_{1}$ is the supremum of all $\delta_{1}$ for which

$$
\liminf _{q \rightarrow \infty} q^{\delta_{1}}\|q \theta\|=0
$$

where $q$ runs through the positive integers. The relationship between these two definitions is that an irrational number $\theta$ is of type $\eta_{1}$ if and only if for every $\tau>\eta_{1}$ there is a constant $c=c(\tau, \theta)$ such that $\theta$ is of type $<\psi$ where $\psi(q)=c q^{\tau-1}$. It is 
well-known that almost all numbers are of type 1 . From Roth's theorem, we note that all algebraic irrationalities $\theta$ satisfy

$$
\left|\theta-\frac{r}{q}\right| \gg \frac{1}{q^{2+\epsilon}}
$$

Therefore all algebraic irrationalities are of type 1 .

Just by using the known average zero-density estimates of $L(s, \chi)$ over all characters of a fixed modulus, we prove

THEOREM 2. Let $\theta$ be of type 1 . Then for every arbitrarily small $\epsilon>0$, unconditionally we have

$$
S(x, \theta) \ll_{\epsilon} x^{c^{*}+\epsilon},
$$

where $c^{*}=\frac{4}{5}$.

We formulate the zero-density hypothesis for all the characters $\chi^{*}$ modulo $(q)$ as :

Average Zero-Density Hypothesis. For $\sigma \geq \frac{1}{2}$, we have,

$$
\sum_{\chi^{*}} N\left(\sigma, T, \chi^{*}\right) \ll(q T)^{2(1-\sigma)}(\log (q T))^{A},
$$

where $A$ is an absolute constant.

Now, we prove

Theorem 3. We assume the Average Zero-Density Hypothesis. Let $\theta$ be of type 1. Then for $\epsilon>0$ arbitrarily small, we have

$$
S(x, \theta) \ll x^{\frac{3}{4}+\epsilon} .
$$

Remark. As we stated earlier, Theorem 1 gives the upper bound for $|S(x, \theta)|$ which depends on the zero density functions. We observe that Theorem A yields the exponent $\frac{3}{4}+\epsilon$ (for all real $\theta$ ) only on the assumption of the Generalised Riemann Hypothesis. The author ${ }^{(2)}$ jointly with M. Ram Murty studied such exponential sums in [13] using a more general form of Vaughan's method. It should be mentioned here that they obtained for example (for $\theta$ of type 1)

$$
S(x, \theta) \ll x^{\frac{4}{5}+\epsilon},
$$

in [13]. The approach of the present paper is entirely different and the results here depend on the zero density functions. As far as Theorem 3 is concerned, we have assumed a weaker hypothesis and obtain a better result when $\theta$ is of type 1 and it seems to be new.

As another interesting application of our Theorem 1, we prove 
THEOREM 4. Let $\chi$ be a character modulo $m$. For every integer $m \geq 1$, we suppose that there exists a constant $g(\geq 2)$ such that

$$
\sum_{\chi(\bmod (m))} N(\sigma, T, \chi) \ll(m T)^{g(1-\sigma)}(\log (m T))^{A},
$$

uniformly for $\frac{1}{2} \leq \sigma \leq 1$. Here $A$ is an absolute constant. If $\theta$ is of type 1 , then we have

$$
S(x, \theta) \ll x^{\nu+\epsilon}
$$

where

$$
\nu=\frac{13}{4}-\frac{3}{g}-\frac{g}{2}
$$

Remark. From the known average zero-density results (see the fundamental work of H.L. Montgomery [8], [9], precisely for example see Theorem 12.1 of [10], see (2.23) and a few subsequent lines of [6]), we find that we can take for example $g=\frac{12}{5}$ in (1.4). We also note that the result of Theorem 4 will be having a better exponent $\nu$ (which is less than $\frac{4}{5}$ ) unconditionally for all $\theta$ of type 1 when $g<\frac{12}{5}$ in (1.4).

\section{Some Lemmas}

Lemma 2.1. Let $\tilde{\beta}=\theta-\frac{r}{q}$ and $q \leq x^{2}$. We have

$$
S(x, \theta)=\sum_{d \mid q} \frac{\mu(d)}{\phi(q / d)} \sum_{\chi(} \chi(r) \tau(\bar{\chi}) S\left(\frac{x}{d}, \chi \chi_{d}, \tilde{\beta} d\right) .
$$

Here $\chi_{d}$ denotes the principal character $(\bmod d)$ and

$$
S\left(y, \chi^{*}, \eta\right)=\sum_{n \leq y} \mu(n) \chi^{*}(n) \mathbf{e}(n \eta)
$$

and

$$
\tau(\bar{\chi})=\sum_{m=1}^{q / d} \bar{\chi}(m) \mathbf{e}\left(\frac{m d}{q}\right) .
$$

Proof. See Lemma 1 of [1].

Remark. The functions $\chi \chi_{d}$ appearing in Lemma 2.1 are characters $(\bmod q)$. For the Gauss sum $\tau(\bar{\chi})$, we need the well-known bound

$$
\tau(\bar{\chi}) \ll\left(\frac{q}{d}\right)^{\frac{1}{2}} .
$$


K. Ramachandra and A. Sankaranarayanan studied (see Theorems 1 and 2 of [12]) certain upper bound estimates "locally" for the function $|\log F(s)|$ (where $F(s)$ is any Dirichlet series satisfying certain general conditions) under the assumption that $F(s)$ is zero-free in a rectangle of the type $\left\{\sigma \geq \frac{1}{2}+\delta, T-H \leq t \leq T+H\right\}$ of $t$ width $2 H$. Here the parameter $H$ could be chosen as small as $H=C \log \log \log T$. We again emphasize here that the Theorems 1 and 2 of [12] (see the appendix of the paper [12]) are more general and they assume neither the Euler product nor any functional equation of the Dirichlet series $F(s)$ concerned. We just record a special case of these general theorems as Lemma 2.2 in the sequel.

Lemma 2.2. Let $\frac{1}{2} \leq \alpha^{*} \leq 1-\delta$. Let $H=C \log \log (q T)$ and $\chi^{*}$ be any Dirichlet character modulo $q$. Suppose the Dirichlet L-function $L\left(s, \chi^{*}\right) \neq 0$ in

$$
\left\{\sigma>\alpha^{*}, T-H \leq t \leq T+H\right\} .
$$

Then for $\left(\alpha^{*}<\sigma \leq 1-\frac{C_{1}}{\log \log (q T)}, T-\frac{H}{2} \leq t \leq T+\frac{H}{2}\right)$, we have :

$$
\left|\log L\left(\sigma+i t, \chi^{*}\right)\right| \leq\left(C_{2}(\log (q T))(\log \log (q T))^{-1}\right) .
$$

Proof. It is a special case of the Theorems 1 and 2 of [12].

Lemma 2.3. Let $m$ be an integer and $1 \leq m \leq x$. We have

$$
\begin{aligned}
S\left(m, \chi^{*}, 0\right) & =\frac{1}{2 \pi i} \int_{2-i T}^{2+i T} \frac{1}{L\left(w, \chi^{*}\right)} \frac{m^{w}}{w} d w+O\left(\frac{m^{2}}{T}\right) \\
& =\frac{1}{2 \pi i} \int_{2-i T}^{2+i T} \frac{1}{L\left(w, \chi^{*}\right)} \frac{m^{w}}{w} d w+O\left(\frac{1}{m}\right),
\end{aligned}
$$

with $T=m^{3}$.

Proof. We apply Lemma 3.12 of [14] with $a_{n}=\mu(n) \chi^{*}(n)$.

Lemma 2.4. Let $\theta$ be of type 1 and $\epsilon>0$ be arbitrarily small. Then for $x$ sufficiently large, there exist integers $r, q$ with $(r, q)=1$ satisfying :

$$
x^{\frac{1}{2(1+\epsilon)}} \leq q \leq x^{\frac{1}{2}}
$$

and

$$
\left|\theta-\frac{r}{q}\right|<x^{-(1-\epsilon)}
$$

Proof. In the sequel, let $\epsilon_{j}>0$ denote fixed but arbitrarily small constants. From the definition of the type 1-property, it follows that for all $\epsilon_{1}>0$, there is a $q_{0}\left(\epsilon_{1}\right)$ such that

$$
\|q \theta\| \geq q^{-\left(1+\epsilon_{1}\right)} \text { for } q \geq q_{0}\left(\epsilon_{1}\right) .
$$


We choose $x$ such that

$$
x^{-\frac{1}{2}}<\min _{q<q_{0}\left(\epsilon_{1}\right)}\|q \theta\| .
$$

By the Dirichlet approximation theorem, there is $\frac{r}{q} \in \mathbb{Q}$ with $(r, q)=1,1 \leq q<x^{\frac{1}{2}}$, such that

$$
\left|\theta-\frac{r}{q}\right|<\frac{1}{q x^{\frac{1}{2}}} \text { which implies that }\|q \theta\|<\frac{1}{x^{\frac{1}{2}}} .
$$

By (2.4.4), we have

$$
q \geq q_{0}\left(\epsilon_{1}\right) .
$$

From (2.4.3) and (2.4.5), we have

$$
q^{-\left(1+\epsilon_{1}\right)} \leq\|q \theta\|<x^{-\frac{1}{2}} \text { which implies that } x^{\frac{1}{2}} \leq q^{\left(1+\epsilon_{1}\right)} .
$$

This means that

$$
q \geq x^{\frac{1}{2\left(1+\epsilon_{2}\right)}} \text { and hence }\left|\theta-\frac{r}{q}\right|<x^{-\left(1-\epsilon_{3}\right)} .
$$

This proves the lemma.

Lemma 2.5. Let $q$ be a positive integer and $d$ be any divisor of $q$. Let $\chi$ denote a character modulo $\left(\frac{q}{d}\right)$ and $\chi_{d}$ denote the principal character modulo $d$. Then for $\sigma \geq \frac{1}{2}$, we have

$$
N\left(\sigma, T, \chi \chi_{d}\right)=N(\sigma, T, \chi) .
$$

Proof. We have (for $\Re s>1$ )

$$
L(s, \chi)=\prod_{p}\left(1-\chi(p) p^{-s}\right)^{-1}
$$

and

$$
L\left(s, \chi \chi_{d}\right)=\prod_{p}\left(1-\chi(p) \chi_{d}(p) p^{-s}\right)^{-1}
$$

We note that

$$
\chi_{d}(p)= \begin{cases}0 & \text { if } p \mid d \\ 1 & \text { if } p \not d\end{cases}
$$

Therefore, we have 


$$
\begin{aligned}
L\left(s, \chi \chi_{d}\right) & =\prod_{p / d}\left(1-\chi(p) p^{-s}\right)^{-1} \prod_{p \mid d}\left(1-\chi(p) \chi_{d}(p) p^{-s}\right)^{-1} \\
& =L(s, \chi) \prod_{p \mid d}\left(1-\chi(p) p^{-s}\right) .
\end{aligned}
$$

We also note that

$$
\prod_{p \mid d}\left(1-\chi(p) p^{-s}\right) \neq 0 \text { for } \Re s>0
$$

Hence we obtain

$$
N\left(\sigma, T, \chi \chi_{d}\right)=N(\sigma, T, \chi)
$$

This proves the lemma.

\section{The construction of the contour $\mathcal{C}$ of integration}

In the following, $C^{*}$ is a generic absolute constant which need not be the same at each occurrence.

Definition: A zero $\rho=: \beta+i \gamma$ of the Dirichlet $L$-function $L\left(s, \chi^{*}\right)$ is said to be $\operatorname{good}$ if $\beta<1-\frac{C^{*}}{(\log \log (q(|t|+2)))}$ and $\rho$ is said to be exceptional otherwise.

Let $T_{0} \leq T\left(\leq x^{3}\right)$ and $x \geq x_{0}$ where $x_{0}$ is sufficiently large. Let $\mathcal{G}$ and $\mathcal{E}$ denote the set of all good and exceptional zeros respectively of all the Dirichlet $L$-functions $L\left(s, \chi^{*}\right)$ with $|\gamma| \leq T$. We denote $|\mathcal{G}|$ and $|\mathcal{E}|$ to mean the cardinality of the sets $\mathcal{G}$ and $\mathcal{E}$ respectively.

Let $\alpha$ be any fixed constant satisfying $\frac{1}{2}+\delta \leq \alpha \leq 1-\delta$ with $\delta$ being any arbitrarily small fixed positive constant. The contour is symmetric with respect to the real axis. So, it suffices to describe it in the upper half-plane. We assume that $|\mathcal{E}|=0$. Therefore, $L\left(s, \chi^{*}\right) \neq 0$ in the region $\left\{\sigma>1-\frac{C^{*}}{(\log \log 2 q(U+10))}, U \leq t \leq 2 U\right\}$ where $C^{*}$ 'is a suitable absolute positive constant and we construct the contour accordingly. We let $T=2^{l_{0}}$. We choose $c$ with $\frac{1}{2} \leq c \leq 1$ such that $H_{0}=c \log \log (q T)=2^{L}$ with a positive integer $L$. For $l \geq L$, put $U=U^{(l)}=2^{l}$. We define the contour for $U<t \leq 2 U$. We let $H=H\left(U^{(l)}\right)=c_{l} \log \log \left(q U^{(l)}\right)$ and choose $c_{l}$ satisfying $\frac{1}{2} \leq c_{l} \leq 1$ such that $\frac{U}{2 H}$ is a positive integer. Then we partition the interval $[U, 2 U]$ into $\frac{U}{2 H}$ disjoint abutting small intervals $I_{j}=I_{j}^{(l)}$ of equal length $2 H$ for $1 \leq j \leq \frac{U}{2 H}$. Let $I_{j}=\left[U_{j}-H, U_{j}+H\right]$ and let

$$
\beta_{j}=\sup \left\{\beta: \rho=\beta+i \gamma, L\left(\rho, \chi^{*}\right)=0, \beta \geq \alpha, \gamma \in\left[U_{j}-2 H, U_{j}+2 H\right]\right\}
$$

and 


$$
\beta_{j}^{*}=\beta_{j}+\frac{C^{*}}{(\log \log 2 q(U+10))} .
$$

We also define (with $\left.H_{0}^{\prime}=H_{0}+2\left(\log \left(q H_{0}\right)\right)^{2}\right)$

$$
\beta_{0}=\sup \left\{\beta: \rho=\beta+i \gamma, L\left(\rho, \chi^{*}\right)=0, \beta \geq \alpha, \gamma \in\left[0,2 H_{0}^{\prime}\right]\right\}
$$

and

$$
\beta_{0}^{*}=\beta_{0}+\frac{C^{*}}{\left(\log \log q H_{0}\right)} .
$$

If there is no zero of $L\left(s, \chi^{*}\right)$ in the rectangle

$$
\left\{\sigma \geq \alpha, U_{j}-2 H \leq t \leq U_{j}+2 H\right\},
$$

then we define $\beta_{j}^{*}=\alpha$. Similar notion applies to $\beta_{0}^{*}$ also.

Thus, the contour $\mathcal{C}$ consists of vertical pieces

$$
V_{j}=:\left\{\begin{array}{c}
{\left[\beta_{j}^{*}+i\left(U_{j}-H+\epsilon\right), \beta_{j}^{*}+i\left(U_{j}+H-\epsilon\right)\right] \text { if } \beta_{j}^{*}<\min \left(\beta_{j-1}^{*}, \beta_{j+1}^{*}\right),} \\
{\left[\beta_{j}^{*}+i\left(U_{j}-H-\epsilon\right), \beta_{j}^{*}+i\left(U_{j}+H+\epsilon\right)\right] \text { if } \beta_{j}^{*}>\max \left(\beta_{j-1}^{*}, \beta_{j+1}^{*}\right),} \\
{\left[\beta_{j}^{*}+i\left(U_{j}-H-\epsilon\right), \beta_{j}^{*}+i\left(U_{j}+H-\epsilon\right)\right]} \\
\quad \text { if } \min \left(\beta_{j-1}^{*}, \beta_{j+1}^{*}\right)<\beta_{j}^{*}<\max \left(\beta_{j-1}^{*}, \beta_{j+1}^{*}\right),
\end{array}\right.
$$

the vertical piece

$$
V_{0}=: \begin{cases}{\left[\beta_{0}^{*}, \beta_{0}^{*}+i\left(H_{0}-\epsilon\right)\right]} & \text { if } \beta_{1}^{*}>\beta_{0}^{*}, \\ {\left[\beta_{0}^{*}, \beta_{0}^{*}+i\left(H_{0}+\epsilon\right)\right]} & \text { if } \beta_{1}^{*}<\beta_{0}^{*}\end{cases}
$$

the horizontal pieces $h_{j}$ with

and

$$
h_{j}(\mathrm{top})=\left[\beta_{j}^{*}+i\left(U_{j}+H-\epsilon\right), \beta_{j+1}^{*}+i\left(U_{j}+H-\epsilon\right)\right]
$$

$$
h_{j}(\text { bottom })=\left[\beta_{j}^{*}+i\left(U_{j}-H+\epsilon\right), \beta_{j-1}^{*}+i\left(U_{j}-H+\epsilon\right)\right]
$$

provided $\beta_{j}^{*}<\min \left(\beta_{j-1}^{*}, \beta_{j+1}^{*}\right)$,

and

$$
h_{j}(\mathrm{top})=\left[\beta_{j+1}^{*}+i\left(U_{j}+H+\epsilon\right), \beta_{j}^{*}+i\left(U_{j}+H+\epsilon\right)\right]
$$

$$
h_{j}(\text { bottom })=\left[\beta_{j-1}^{*}+i\left(U_{j}-H-\epsilon\right), \beta_{j}^{*}+i\left(U_{j}-H-\epsilon\right)\right]
$$

provided $\beta_{j}^{*}>\max \left(\beta_{j-1}^{*}, \beta_{j+1}^{*}\right)$,

and

$$
h_{j}(\mathrm{top})=\left[\beta_{j}^{*}+i\left(U_{j}+H-\epsilon\right), \beta_{j+1}^{*}+i\left(U_{j}+H-\epsilon\right)\right]
$$




$$
h_{j}(\text { bottom })=\left[\beta_{j-1}^{*}+i\left(U_{j}-H-\epsilon\right), \beta_{j}^{*}+i\left(U_{j}-H-\epsilon\right)\right]
$$

provided $\beta_{j-1}^{*}<\beta_{j}^{*}<\beta_{j+1}^{*}$,

and

$$
h_{j}(\mathrm{top})=\left[\beta_{j+1}^{*}+i\left(U_{j}+H-\epsilon\right), \beta_{j}^{*}+i\left(U_{j}+H-\epsilon\right)\right]
$$

$$
h_{j}(\text { bottom })=\left[\beta_{j}^{*}+i\left(U_{j}-H-\epsilon\right), \beta_{j-1}^{*}+i\left(U_{j}-H-\epsilon\right)\right]
$$

provided $\beta_{j+1}^{*}<\beta_{j}^{*}<\beta_{j-1}^{*}$, and the similar horizontal pieces $h_{0, l}$ (this is just a notation only) that link the top (respectively the bottom) vertical pieces of the ranges

$$
U^{(l-1)} \leq t \leq 2 U^{(l-1)} \quad \text { (respectively) } U^{(l)} \leq t \leq 2 U^{(l)} .
$$

\section{The treatment of $S\left(y, \chi^{*}, \eta\right)$}

We keep in mind that the modulus $q$ satisfies the inequality $q \geq q_{0}$ and there is no exceptional zero in the following.

We have

$$
S\left(y, \chi^{*}, \eta\right)=\sum_{m=1}^{y-1} S\left(m, \chi^{*}, 0\right) \mathbf{e}(\eta m)(1-\mathbf{e}(\eta))+S\left(y, \chi^{*}, 0\right) \mathbf{e}(\eta y) .
$$

By Lemma 2.3, we have (with $T=m^{3}\left(\leq y^{3} \leq\left(\frac{x}{d}\right)^{3} \leq x^{3}\right)$

$$
\begin{aligned}
\sum_{m=1}^{y-1} S\left(m, \chi^{*}, 0\right) \mathbf{e}(\eta m) & =\frac{1}{2 \pi i} \int_{2-i T}^{2+i T} \frac{1}{L\left(w, \chi^{*}\right) w}\left(\sum_{m=1}^{y-1} m^{w} \mathbf{e}(\eta m)\right) d w+O(\log y) \\
& =I^{*}+O(\log y) \quad \text { (say). }
\end{aligned}
$$

We now replace the path $[2-i T, 2+i T]$ by the contour $\mathcal{C}$ constructed in section 3 .

By Lemma 2.2 (for large $|t|\left(\geq H_{0}\right)$ ), we have

$$
\left|L\left(\sigma+i t, \chi^{*}\right)\right|^{-1} \ll(q U)^{\epsilon}
$$

for $\sigma+i t \in V_{j}$ and $\sigma+i t \in h_{j}$. The horizontal slab with $|t| \in\left[0, H_{0}\right]$ is treated as follows. We redefine

$$
\beta_{0}^{*}=\left\{\begin{array}{lll}
\beta_{0,1}^{*} & \text { if } & |t| \in\left[0,2^{7}\right] \\
\beta_{0,2}^{*} & \text { if } & |t| \in\left[2^{3}, H_{0}^{\prime}\right] .
\end{array}\right.
$$

For the portion $|t| \in\left[2^{5}, H_{0}\right]$, we first observe that the region

$$
\left\{10 \geq \sigma \geq \beta_{0,2}^{*} \geq \beta_{0}+\frac{C^{*}}{\left(\log \log q H_{0}\right)}, 2^{3} \leq|t| \leq H_{0}^{\prime}\right\}
$$


is zero-free for $L\left(s, \chi^{*}\right)$ and hence applying Borel-Caratheodary theorem, we get (for $\left.10 \geq \sigma \geq \beta_{0}+\frac{C^{*}}{\left(\log \log q H_{0}\right)}\right)$

$$
\left|\log L\left(\sigma+i t, \chi^{*}\right)\right| \ll\left(\log \left(q H_{0}\right)\right)^{1-\epsilon} \ll(\log (q T))^{1-\epsilon},
$$

and so the above estimate holds when $\beta_{0,2}^{*}+i t \in V_{0}$ with $t \in\left[2^{5}, H_{0}\right]$ provided $T \geq T_{0}$ (where $T_{0}$ is sufficiently large).

The portion $|t| \in\left[0,2^{5}\right]$ is dealt as follows. Note that by our assumption, $L\left(s, \chi^{*}\right)$ does not have any exceptional zero. We first observe that the region

$$
\left\{10 \geq \sigma \geq 1-\frac{C^{* *}}{2(\log \log q)} \geq \beta_{0,1}^{*}, \quad|t| \leq 2^{7}\right\}
$$

is zero-free for $L\left(s, \chi^{*}\right)$ with a suitable absolute constant $C^{* *}$. If $\sigma \geq 1-\frac{C^{* *}}{2(\log \log q)}$, then we have

$$
\left|L\left(s, \chi^{*}\right)\right| \ll(\log q)^{1-\epsilon} .
$$

If $|s-1| \leq \frac{C^{* *}}{10(\log \log q)}$, then we observe that

$$
\begin{aligned}
\left|L\left(s, \chi^{*}\right)\right| & \leq\left|L\left(s, \chi^{*}\right)-L\left(1, \chi^{*}\right)\right|+\left|L\left(1, \chi^{*}\right)\right| \\
& \ll|(s-1)|(\log q)^{1-\epsilon}+\log q \\
& \ll(\log q) .
\end{aligned}
$$

Now, we apply the Borel-Caratheodary Theorem suitably to the function $\log \left((s-1) L\left(s, \chi^{*}\right)\right)$ with concentric circles having centre at $2+i t$ and radii $\frac{1}{2}, 1+$ $\frac{C^{* *}}{2(\log \log q)}$ and conclude that

$$
\left|\log L\left(\beta_{0,1}^{*}+i t, \chi^{*}\right)\right| \ll\left|\log \left(\frac{1}{1-\beta_{0,1}^{*}}\right)\right|+(\log q)^{1-\epsilon} \ll(\log (q T))^{1-\epsilon},
$$

for $T \geq T_{0}$. This implies that

$$
\left|L\left(\beta_{0,1}^{*}+i t, \chi^{*}\right)\right|^{-1} \ll(q T)^{\epsilon}
$$

whenever $|t| \in\left[0,2^{5}\right]$.

By partial summation, we obtain

$$
\sum_{m=1}^{y-1} m^{\beta_{j}^{*}+i t} \mathbf{e}(\eta m)=(y-1)^{\beta_{j}^{*}} \sum_{m \leq y-1} m^{i t} \mathbf{e}(\eta m)-\beta_{j}^{*} \int_{1}^{y-1}\left(\sum_{m \leq u} m^{i t} \mathbf{e}(\eta m)\right) u^{\beta_{j}^{*}-1} d u .
$$

From (4.3) and (4.9), we observe that the contribution of the vertical path $V_{j}$ to $I^{*}$ is 


$$
\begin{aligned}
I^{*}\left(V_{j}\right) & =: \sum_{m=1}^{y-1} \frac{1}{2 \pi i} \int_{V_{j}} \frac{1}{L\left(w, \chi^{*}\right)} \frac{m^{w}}{w} \mathbf{e}(\eta m) d w \\
& \ll H U^{-1}(q U)^{\epsilon} y^{\beta_{j}^{*}} \max _{u \leq y} \sup _{t \in[U, 2 U]}\left|\sum_{m \leq u} m^{i t} \mathbf{e}(\eta m)\right|,
\end{aligned}
$$

an analogous estimate

$$
\begin{aligned}
I^{*}\left(V_{0}\right) & \ll H_{0}(q T)^{\epsilon} y^{\beta_{0}^{*} \times} \\
& \times\left\{\max _{u \leq y} \sup _{t \in\left[0,2^{5}\right]}\left|\sum_{m \leq u} m^{i t} \mathbf{e}(\eta m)\right|+\max _{u \leq y} \sup _{t \in\left[2^{5}, H_{0}\right]}\left|\sum_{m \leq u} m^{i t} \mathbf{e}(\eta m)\right|\right\},
\end{aligned}
$$

and the contribution of the horizontal path $h_{j}$ to $I^{*}$ is

$$
\begin{aligned}
I^{*}\left(h_{j}\right) & =: \sum_{m=1}^{y-1} \frac{1}{2 \pi i} \int_{h_{j}} \frac{1}{L\left(w, \chi^{*}\right)} \frac{m^{w}}{w} \mathbf{e}(\eta m) d w \\
& \ll U^{-1}(q U)^{\epsilon} y^{\beta_{j}^{*}} \max _{u \leq y} \sup _{t \in[U, 2 U]}\left|\sum_{m \leq u} m^{i t} \mathbf{e}(\eta m)\right| .
\end{aligned}
$$

For the horizontal pieces $h_{0, l}$, an analogous estimate applies.

\section{Zero density functions}

We presume that $|\mathcal{E}|=0$. As before, we let $\alpha$ be any fixed constant satisfying $\frac{1}{2}+\delta \leq \alpha \leq 1-\delta$ with $\delta$ being any arbitrarily small fixed positive constant. We now choose a partition of the interval $[\alpha, 1]$ namely

$$
\alpha=\alpha_{0}<\alpha_{1}<\cdots<\alpha_{j-1}<\alpha_{j}=1
$$

with

$$
\alpha_{j}-\alpha_{j-1}<\epsilon
$$

The number of $j$ - values for which $\beta_{j}^{*} \in\left[\alpha_{l-1}, \alpha_{l}\right]$ is bounded by $N\left(\alpha_{l-1}, 2 U, \chi^{*}\right)$. From (4.10) and (4.11), we observe that the total contribution from all the vertical bits and the horizontal bits to the integral $I^{*}$ (for $U \leq t \leq 2 U$ ) is 


$$
\begin{aligned}
C\left(I^{*}\right) & =: \sum_{j}\left(I^{*}\left(V_{j}\right)+I^{*}\left(h_{j}\right)\right) \\
& \ll H U^{-1}(q U)^{\epsilon}\left(\max _{u \leq y} \sup _{t \in[U, 2 U]}\left|\sum_{m \leq u} m^{i t} \mathbf{e}(\eta m)\right|\right)\left(\sum_{j} y^{\beta_{j}^{*}}\right) \\
& \ll H U^{-1}(q U)^{\epsilon}\left(\max _{u \leq y} \sup _{t \in[U, 2 U]}\left|\sum_{m \leq u} m^{i t} \mathbf{e}(\eta m)\right|\right)\left(U y^{\alpha+\epsilon}+\int_{\alpha}^{1} y^{\sigma+\epsilon} N\left(\sigma, 2 U, \chi^{*}\right) d \sigma\right),
\end{aligned}
$$

since $\beta_{j}^{*}>\beta_{j}$.

Thus we arrive at,

$$
\begin{aligned}
S^{*} & =: \sum_{m=1}^{y-1} S\left(m, \chi^{*}, 0\right) \mathbf{e}(\eta m) \\
& \ll \sum_{\substack{L \leq j \leq l_{0}, U=2^{j}}}\left\{H U^{-1}(q U)^{\epsilon} \max _{u \leq y} \sup _{t \in[U, 2 U]}\left|\sum_{m \leq u} m^{i t} \mathbf{e}(\eta m)\right|\left(U y^{\alpha+\epsilon}+\int_{\alpha}^{1} y^{\sigma+\epsilon} N\left(\sigma, 2 U, \chi^{*}\right) d \sigma\right)\right\} \\
& +H_{0}(q T)^{\epsilon}\left\{\max _{u \leq y} \sup _{t \in\left[0,2^{5}\right]}\left|\sum_{m \leq u} m^{i t} \mathbf{e}(\eta m)\right|+\max _{u \leq y} \sup _{t \in\left[2^{5}, H_{0}\right]}\left|\sum_{m \leq u} m^{i t} \mathbf{e}(\eta m)\right|\right\} \times \\
& \times\left(2^{L} y^{\alpha+\epsilon}+2^{L} \int_{\alpha}^{1} y^{\sigma+\epsilon} \frac{N\left(\sigma, 2^{L}, \chi^{*}\right)}{2^{L}} d \sigma\right)+\log y \\
& \ll H(q T)^{4 \epsilon} \max _{u \leq y}\left(\sup _{t \in[0,2]}\left|\sum_{m \leq u} m^{i t} \mathbf{e}(\eta m)\right|+\sum_{2 \leq j \leq l_{0}}\left|\sup _{t \in\left[2^{j-1}, 2^{j}\right]}\right| \sum_{m \leq u} m^{i t} \mathbf{e}(\eta m) \mid\right) \times \\
& \times\left(y^{\alpha+\epsilon}+\int_{\alpha}^{1} y^{\sigma+\epsilon} \frac{N\left(\sigma, T, \chi^{*}\right)}{T} d \sigma\right)+\log y \\
& \ll \max _{1 \leq T \leq y^{3}} T^{-1}(q T)^{5 \epsilon} \max _{u \leq y}\left(\sup _{t \in[0,2]}\left|\sum_{m \leq u} m^{i t} \mathbf{e}(\eta m)\right|+\sum_{2 \leq j \leq l_{0}} \sup _{t \in\left[2^{j-1}, 2^{j}\right]}\left|\sum_{m \leq u} m^{i t} \mathbf{e}(\eta m)\right|\right) \times \\
(5.3) & \times\left(T y^{\alpha+\epsilon}+\int_{\alpha}^{1} y^{\sigma+\epsilon} N\left(\sigma, T, \chi^{*}\right) d \sigma\right)+\log y .
\end{aligned}
$$

We treat $S\left(y, \chi^{*}, 0\right) \mathbf{e}(\eta y)$ in a similar fashion and obtain 


$$
S\left(y, \chi^{*}, 0\right) \mathbf{e}(\eta y) \ll \max _{1 \leq T \leq y^{3}} T^{-1}(q T)^{5 \epsilon}\left(T y^{\alpha+\epsilon}+\int_{\alpha}^{1} y^{\sigma+\epsilon} N\left(\sigma, T, \chi^{*}\right) d \sigma\right)+\log y .
$$

Let $t^{*}$ be the value at which the quantity $\left|\sum_{m \leq u} m^{i t} \mathbf{e}(\eta m)\right|$ attains its supremum. We just estimate this sum trivially and get

$$
\sup _{t \in\left[0,2^{l_{0}}\right]}\left|\sum_{m \leq u} m^{i t} \mathbf{e}(\eta m)\right| \leq u \leq|\eta|^{-1}(1+|\eta| u)
$$

Remark. Any non-trivial bound to the sum in the left hand side of (5.5) only in terms of $u$ will surely sharpen Theorem 1 .

\section{Proof of the Theorems}

Proof of Theorem 1. We take $\chi^{*}=\chi \chi_{d}$ which are characters modulo $q$ for various $\chi$ modulo $\frac{q}{d}$. Here $\chi_{d}$ denotes the principal character modulo $d$. We fix our $\eta=\tilde{\beta} d$. The proof follows on substituting the estimates obtained in (5.3), (5.4) and (5.5) in Lemma 2.1 appropriately. Thus using the estimates (5.3), (5.4) and (5.5), we first obtain

$$
\begin{aligned}
S\left(y, \chi^{*}, \eta\right) & \ll \max _{1 \leq T \leq y^{3}}(1+y|\eta|) T^{-1}(q T)^{10 \epsilon}\left(T y^{\alpha+\epsilon}+\int_{\alpha}^{1} y^{\sigma+\epsilon} N\left(\sigma, T, \chi^{*}\right) d \sigma\right) \\
& +\log y \\
& \ll \max _{1 \leq T \leq y^{3}}(1+y|\eta|) T^{-1}(q T)^{10 \epsilon}\left(T y^{\alpha+\epsilon}+\int_{\frac{1}{2}}^{1} y^{\sigma+\epsilon} N\left(\sigma, T, \chi^{*}\right) d \sigma\right),
\end{aligned}
$$

provided $|\mathcal{E}|=0$. Thus, the set $\mathcal{G}$ contributes to $\sum_{\chi\left(\bmod \frac{q}{d}\right)} S\left(y, \chi^{*}, \eta\right)$ precisely an amount which is in absolute value

$$
\ll \sum_{\chi\left(\frac{q}{d}\right)}\left|S\left(y, \chi^{*}, \eta\right)\right|
$$

and from (6.1) and Lemma 2.1 (with $y=\frac{x}{d}$ ), we observe that this leads to the second sum term (appearing in the right hand side of (1.3)) over all the divisors $d$ of $q$ with maximum over $T$ of certain integral involving the zero density functions provided the set $\mathcal{E}$ is empty.

The case $|\mathcal{E}| \geq 1$ is dealt as follows. From the density estimates (see for example (6.4) and (6.5)), we have (for $\frac{1}{2} \leq \sigma \leq 1$ ) 


$$
\sum_{\chi^{*}} N\left(\sigma, T, \chi^{*}\right) \ll(q T)^{\frac{12}{5}(1-\sigma)}(\log (q T))^{A}
$$

with an absolute constant $A$. Therefore we have,

$$
\begin{aligned}
|\mathcal{E}| & \ll \sum_{\chi^{*}} N\left(1-\frac{C^{*}}{(\log \log (q T))}, T, \chi^{*}\right) \\
& \ll \exp \left(C_{1}^{*}\left(\frac{\log (q T)}{\log \log (q T)}\right)\right) \\
& \ll(q T)^{\epsilon} .
\end{aligned}
$$

For each of these exceptional zero from the set $\mathcal{E}$, we estimate $S\left(y, \chi^{*}, \eta\right)$ trivially and from Lemma 2.1 (with $y=\frac{x}{d}$ ), we observe that the contribution coming from the set $\mathcal{E}$ to $S(x, \theta)$ is in absolute value

$$
\ll q^{-\frac{1}{2}+\epsilon}(q T)^{\epsilon} x \ll q^{-\frac{1}{2}} x^{1+10 \epsilon},
$$

since, $q \leq x^{2}, T \leq x^{3}$. This leads to the first term appearing in the right hand side of (1.3). This proves Theorem 1 .

Proof of Theorem 2. The fundamental work on zero density estimates of Montgomery (see [8] and [9]) and the methods of [5] and Theorem 1 of [6] (for example see (2.23) and a few subsequent lines of [6]) enable one to deduce the following zero density theorem (which is a variation of Theorem 12.1 of [10]).

Average Zero-Density Theorem. Suppose that $q \geq 1$ and $T \geq 2$. Let $\chi^{*}$ denote characters modulo $q$. For $\frac{1}{2} \leq \sigma \leq \frac{3}{4}+\epsilon$, we have

$$
\sum_{\chi^{*}} N\left(\sigma, T, \chi^{*}\right) \ll(q T)^{\frac{3(1-\sigma)}{2-\sigma}}(\log (q T))^{9}\left(\ll(q T)^{\frac{12}{5}(1-\sigma)}(\log (q T))^{9}\right),
$$

and for $\frac{3}{4}+\epsilon \leq \sigma \leq 1$, we have

$$
\sum_{\chi^{*}} N\left(\sigma, T, \chi^{*}\right) \ll(q T)^{\frac{12}{5}(1-\sigma)}(\log (q T))^{A} .
$$

Let $f_{1}(\sigma)=: \sigma+\frac{3(1-\sigma)}{2(2-\sigma)}$. Then, $f_{1}^{(1)}(\sigma)=1-\frac{3}{2(2-\sigma)^{2}}$. If $\sigma_{0}=2-\left(\frac{3}{2}\right)^{\frac{1}{2}}$, then $f_{1}^{(1)}\left(\sigma_{0}\right)=0$ and $f_{1}^{(2)}\left(\sigma_{0}\right)<0$. We notice that the interval $\left[\frac{1}{2}, \frac{3}{4}+\epsilon\right]$ is contained in the interval $\left[\frac{1}{2}, 2-\left(\frac{3}{2}\right)^{\frac{1}{2}}\right]$. We find that $f_{1}^{(1)}\left(\frac{1}{2}\right)>0$ and hence the function $f_{1}(\sigma)$ is increasing in the interval $\left[\frac{1}{2}, \frac{3}{4}+\epsilon\right]$. Therefore

$$
\max _{\frac{1}{2} \leq \sigma \leq \frac{3}{4}+\epsilon} f_{1}(\sigma)=f_{1}\left(\frac{3}{4}+\epsilon\right) \leq \frac{21}{20}+5 \epsilon .
$$


If $f_{2}(\sigma)=: \sigma+\frac{6}{5}(1-\sigma)$, then we find that (for $\left.\frac{3}{4}+\epsilon \leq \sigma \leq 1\right)$

$$
f_{2}(\sigma) \leq \frac{21}{20}+5 \epsilon .
$$

We fix our $\alpha=\frac{1}{2}+\delta$ in Theorem 1 where $\delta$ is any arbitrarily small positive constant.

Hence from Theorem 1, Lemmas 2.4 and 2.5, we have

$$
\begin{aligned}
S & =: S(x, \theta) \\
& \ll q^{-\frac{1}{2}} x^{1+10 \epsilon}+\sum_{d \mid q}\left(\frac{q}{d}\right)^{\frac{1}{2}} \cdot \frac{1}{\phi\left(\frac{q}{d}\right)}\left(1+x\left|\theta-\frac{r}{q}\right|\right) \times
\end{aligned}
$$

$$
\times\left(\max _{1 \leq T \leq\left(\frac{x}{d}\right)^{3}} T^{-1}(q T)^{10 \epsilon} \int_{\frac{1}{2}}^{1}\left(T \phi\left(\frac{q}{d}\right)\left(\frac{x}{d}\right)^{\alpha+\epsilon}+\left(\frac{x}{d}\right)^{\sigma+\epsilon}\left(\sum_{\chi} N(\sigma, T, \chi)\right)\right) d \sigma\right)
$$

with $q \geq x^{\frac{1}{2(1+\epsilon)}}$ and $\left|\theta-\frac{r}{q}\right|<x^{-(1-\epsilon)}$. Now, we split the integral into two integrals, one in which the range of integration varies from $\frac{1}{2}$ to $\frac{3}{4}+\epsilon$ whereas the range in the other varies from $\frac{3}{4}+\epsilon$ to 1 .

Therefore from (6.4), (6.5) and (6.8), we get

$$
S(x, \theta) \ll x^{25 \epsilon}\left(Q_{0}+Q_{1}+Q_{2}+Q_{3}\right),
$$

where

$$
\begin{gathered}
Q_{0} \ll x^{\frac{3}{4}} ; Q_{1}=: \max _{d \leq x^{\frac{1}{2}}} \frac{x^{\frac{3}{4}+\delta+\epsilon}}{d^{1+\delta}} \\
Q_{2}=: \max _{d \leq x^{\frac{1}{2}}} \max _{1 \leq T \leq\left(\frac{x}{d}\right)^{3}} \max _{\frac{1}{2} \leq \sigma \leq \frac{3}{4}+\epsilon} T^{-1}\left(\frac{x^{\frac{1}{2}}}{d}\right)^{-\frac{1}{2}}\left(\frac{x}{d}\right)^{\sigma+\epsilon}\left(\frac{x^{\frac{1}{2}} T}{d}\right)^{\frac{3(1-\sigma)}{2-\sigma}}
\end{gathered}
$$

and

$$
Q_{3}=: \max _{d \leq x^{\frac{1}{2}}} \max _{1 \leq T \leq\left(\frac{x}{d}\right)^{3}} \max _{\frac{3}{4}+\epsilon \leq \sigma \leq 1} T^{-1}\left(\frac{x^{\frac{1}{2}}}{d}\right)^{-\frac{1}{2}}\left(\frac{x}{d}\right)^{\sigma+\epsilon}\left(\frac{x^{\frac{1}{2}} T}{d}\right)^{\frac{12(1-\sigma)}{5}} .
$$

We observe that the expressions on the right hand side of (6.10), (6.11) and (6.12) above (without maximums) are decreasing in the variables $d$ and $T$. Therefore the maximum is attained for $d=1$ and $T=1$. Hence, it is easy to see that 


$$
\begin{gathered}
Q_{0} \ll x^{\frac{3}{4}} ; Q_{1} \ll x^{\frac{3}{4}+10 \delta+\epsilon} \\
Q_{2} \ll \max _{\frac{1}{2} \leq \sigma \leq \frac{3}{4}+\epsilon} x^{\sigma+\epsilon+\frac{3(1-\sigma)}{2(2-\sigma)}-\frac{1}{4}} \ll x^{\frac{4}{5}+5 \epsilon}
\end{gathered}
$$

and

$$
Q_{3} \ll \max _{\frac{3}{4}+\epsilon \leq \sigma \leq 1} x^{\sigma+\epsilon+\frac{6(1-\sigma)}{5}-\frac{1}{4}} \ll x^{\frac{4}{5}+5 \epsilon} .
$$

We note that

$$
c^{*}=\frac{4}{5} .
$$

Thus, from (6.13), (6.14), (6.15) and (6.9), we obtain

$$
S(x, \theta) \ll x^{c^{*}+50 \epsilon+10 \delta} .
$$

Since $\epsilon$ and $\delta$ are arbitrarily small positive constants, the proof of Theorem 2 is complete.

Proof of Theorem 3. As in the proof of Theorem 2, we now choose our $\alpha$ here to be $\alpha=\frac{1}{2}+\delta$ in Theorem 1 so that (for $\theta$ of type 1 ) we have

$$
\begin{aligned}
S & =: S(x, \theta) \\
& \ll q^{-\frac{1}{2}} x^{1+10 \epsilon}+\sum_{d \mid q}\left(\frac{q}{d}\right)^{\frac{1}{2}} \cdot \frac{1}{\phi\left(\frac{q}{d}\right)}\left(1+x\left|\theta-\frac{r}{q}\right|\right) \times \\
& \times\left(\max _{1 \leq T \leq\left(\frac{x}{d}\right)^{3}} T^{-1}(q T)^{10 \epsilon}\left\{\int_{\frac{1}{2}}^{1}\left(T \phi\left(\frac{q}{d}\right)\left(\frac{x}{d}\right)^{\alpha+\epsilon}+\left(\frac{x}{d}\right)^{\sigma+\epsilon}\left(\sum_{\chi \bmod \left(\frac{q}{d}\right)} N(\sigma, T, \chi)\right)\right) d \sigma\right\}\right) \\
& \ll q^{-\frac{1}{2}} x^{1+10 \epsilon}+x^{25 \epsilon} \max _{d \leq x^{\frac{1}{2}}}\left(\frac{x^{\frac{1}{2}}}{d}\right)^{\frac{1}{2}}\left(\frac{x^{\frac{1}{2}}}{d}\right)^{-1}\left(1+x^{\epsilon}\right) \times \\
& \left.\times\left(\left(\max _{1 \leq T \leq\left(\frac{x}{d}\right)^{3}}\left(\left(\frac{x^{\frac{1}{2}}}{d}\right)\left(\frac{x}{d}\right)^{\frac{1}{2}+\delta+\epsilon}+T^{-1} \max _{\frac{1}{2} \leq \sigma \leq 1}\left(\frac{x}{d}\right)^{\sigma+\epsilon}\left(\frac{x^{\frac{1}{2}}}{d} T\right)^{2(1-\sigma)+\epsilon}\right)\right)\right)\right) \\
& \ll x^{\frac{3}{4}+20 \epsilon}
\end{aligned}
$$

$$
+x^{-\frac{1}{4}+30 \epsilon}\left\{\max _{d \leq x^{\frac{1}{2}}}\left(\frac{x^{1+10 \delta+\epsilon}}{d}\right)+\max _{d \leq x^{\frac{1}{2}}} \max _{1 \leq T \leq\left(\frac{x}{d}\right)^{3}} \max _{\frac{1}{2} \leq \sigma \leq 1} d^{\frac{1}{2}} T^{-1}\left(\frac{x}{d}\right)^{\sigma+\epsilon}\left(\frac{x^{\frac{1}{2}}}{d} T\right)^{2(1-\sigma)+\epsilon}\right\} .
$$


Observing the monotonicity in $d$ and $T$, we see that the maximum is attained for $d=1$ and $T=1$. Thus we obtain

$$
S(x, \theta) \ll x^{\frac{3}{4}+50 \epsilon+10 \delta} .
$$

Since $\epsilon$ and $\delta$ are arbitrarily small positive constants, this completes the proof of Theorem 3.

Proof of Theorem 4. From the known density estimates (see Theorem 12.1 of [10], the methods of [5] and Theorem 1 of [6]), we can very well use the bound

$$
\sum_{\chi\left(\bmod \frac{q}{d}\right)} N(\sigma, T, \chi) \ll \begin{cases}\left(\frac{q T}{d}\right)^{\frac{3(1-\sigma)}{2-\sigma}}\left(\log \left(\frac{q T}{d}\right)\right)^{A} & \text { if } \frac{1}{2} \leq \sigma \leq 2-\frac{3}{g}, \\ \left(\frac{q T}{d}\right)^{g(1-\sigma)}\left(\log \left(\frac{q T}{d}\right)\right)^{A} & \text { if } 2-\frac{3}{g} \leq \sigma \leq 1,\end{cases}
$$

for $2 \leq g \leq \frac{12}{5}$ with a suitable absolute constant $A$. We observe that we can split the integral in (6.8) into two integrals, one in which the range of integration runs from $\frac{1}{2}$ to $2-\frac{3}{g}$ and for the other, the range of integration runs from $2-\frac{3}{g}$ to 1 . We use the first estimate of (6.18) when $\sigma$ varies in the interval $\left[\frac{1}{2}, 2-\frac{3}{g}\right]$ whereas we use the second estimate of (6.18) when $\sigma$ varies in the interval $\left[2-\frac{3}{g}, 1\right]$. We note that $f_{1}(\sigma)=: \sigma+\frac{3(1-\sigma)}{2(2-\sigma)}$ is an increasing function throughout in the interval $\left[\frac{1}{2}, 2-\frac{3}{g}\right]$. Therefore, we have

$$
-\frac{1}{4}+\left(\max _{\frac{1}{2} \leq \sigma \leq 2-\frac{3}{g}} f_{1}(\sigma)\right) \leq \frac{13}{4}-\frac{3}{g}-\frac{g}{2}+\epsilon
$$

and

$$
-\frac{1}{4}+\left(\max _{2-\frac{3}{g} \leq \sigma \leq 1} \sigma+\frac{g}{2}(1-\sigma)\right) \leq \frac{13}{4}-\frac{3}{g}-\frac{g}{2}+\epsilon .
$$

Thus, we obtain (for $\theta$ of type 1)

$$
S(x, \theta) \ll x^{c^{* *}+\epsilon}
$$

where

$$
c^{* *}=\max \left(\frac{3}{4}, \frac{13}{4}-\frac{3}{g}-\frac{g}{2}\right)=\frac{13}{4}-\frac{3}{g}-\frac{g}{2}=\nu,
$$

since $\frac{12}{5} \geq g \geq 2$. We observe that whenever $g<\frac{12}{5}$ we obtain a better exponent $c^{* *}$ which is strictly less than $\frac{4}{5}$ provided $\theta$ is of type 1 . 


\section{Concluding Remarks}

If $\theta$ is of type $\eta_{1}(\geq 1)$, then we have

$$
\|q \theta\| \geq q^{-\left(\eta_{1}+\epsilon\right)} \text { for } q \geq q_{0}(\epsilon)
$$

and hence we do have (as in Lemma 2.4)

$$
q \geq x^{\frac{1}{2 \eta_{1}}-\epsilon} \text { and so }\left|\theta-\frac{r}{q}\right|<\frac{1}{q x^{\frac{1}{2}}}<x^{-\left(\frac{1}{2}+\frac{1}{2 \eta_{1}}-\epsilon\right)} .
$$

Therefore, the proof of Theorem 2 essentially leads to :

ConCLUSION 1. Let $\eta_{1}$ be a real number satisfying $1 \leq \eta_{1}<\frac{1}{2 c^{*}-1}$. Let $\theta$ be of type $\eta_{1}$. Then for every arbitrarily small $\epsilon>0$, we have

$$
S(x, \theta) \ll x^{c^{*}+\frac{1}{2}-\frac{1}{2 \eta_{1}}+\epsilon}
$$

where $c^{*}$ is the same positive constant as in Theorem 2

and the proof of Theorem 3 leads to

Conclusion 2. Let $\eta_{1}$ be a real number satisfying $1 \leq \eta_{1}<2$. Let $\theta$ be of type $\eta_{1}$. If we assume the Average Zero- Density Hypothesis, then for every arbitrarily small $\epsilon>0$, we have

$$
S(x, \theta) \ll x^{\frac{5}{4}-\frac{1}{2 \eta_{1}}+\epsilon} .
$$

From a theorem of Walfisz (see [15]), we have unconditionally

$$
M(x)=: S(x, 0) \ll x \exp \left(-C(\log x)^{\frac{3}{5}}(\log \log x)^{-\frac{1}{5}}\right)
$$

and this is the best unconditional result available till today (when $\theta=0$ ). It is a well-known fact that The Riemann Hypothesis implies that

$$
M(x)=: S(x, 0) \ll x^{\frac{1}{2}+\epsilon}
$$

for any small positive constant $\epsilon$.

However, in view of Theorem A (as mentioned earlier in remark 1 of Theorem 2), Even on the assumption of the Generalised Riemann Hypothesis, all that we can conclude is that (for all real $\theta$ )

$$
S(x, \theta) \ll x^{\frac{3}{4}+\epsilon} .
$$

It should be mentioned here that the Theorem 1 is powerful and yields definitely a very nice non-trivial upper bound for $|S(x, \theta)|$ unconditionally whenever we know the type of $\theta$ as mentioned in the conclusion 1 (i.e for a certain type of irrational numbers $\theta$ ) and conditionally as mentioned in the conclusion 2. However, we must also note that the Theorem 1 may yield only the trivial upper bound for $|S(x, \theta)|$ for example 
when $\theta=0$ (or $\theta$ being any rational integer for that matter) and it looks to be a disadvantage of Theorem 1.

Acknowledgement : This project was carried out and completed when the author ${ }^{(2)}$ visited the Department for Number Theory and Probability Theory, University of Ulm, Germany in 2004 and he wishes to thank the University of Ulm for its warm hospitality and generous support. The authors are thankful to Professor R. Balasubramanian for significant suggestions and the anonymous referee for some fruitful comments.

\section{REFERENCES}

[1] R.C. Baker and G. Harman, Exponential sums formed with the Möbius function, J. London Math. Soc., (2) 43 (1991), 193-198.

[2] H. Davenport, On some infinite series involving arithmetical functions-II, Quart. J. Math., 8 (1937), 313-320.

[3] H. Davenport, Multiplicative number theory, 2nd edition, revised by H.L. Montgomery (Springer, New York, 1980).

[4] D. Hajela and B. Smith, On the maximum of an exponential sum of the Möbius function, Lecture Notes in Mathematics, 1240 (Springer, Berlin, 1987), 145-164.

[5] M.N. Huxley, On the difference between consecutive primes, Invent. Math., 15 (1972), 164-170.

[6] M.N. Huxley, Large values of Dirichlet polynomials, III, Acta Arith., 26 (1975), 435-444.

[7] L. Kuipers and H. Niederreiter, Uniform distributions of sequences, Wiley, (1974).

[8] H.L. Montgomery, Mean and large values of Dirichlet polynomials, Invent. Math., 8 (1969), 334-345.

[9] H.L. Montgomery, Zeros of L- functions, Invent. Math., 8 (1969), 346-354.

[10] H.L. Montgomery, Topics in multiplicative number theory, Lecture Notes in Mathematics, 227 (Springer, Berlin, 1971).

[11] H.L. Montgomery and R.C. Vaughan, Exponential sums with multiplicative coefficients, Invent. Math., 43 (1977), 69-82.

[12] K. Ramachandra and A. Sankaranarayanan, On some theorems of Littlewood and Selberg-III, Ann. Acad. Sci. Fenn. Ser. A I Math., 16 (1991), 139-149.

[13] M. Ram Murty and A. Sankaranarayanan, Averages of exponential twists of the Liouville function, Forum Math., 14 (2002), no: 2, 273-291.

[14] E.C. Titchmarsh, The theory of the Riemann zeta-function, 2nd Edition ( edited by D.R. Heath-Brown ), Clarendon Press ( Oxford ) (1986).

[15] A. Walfisz, Weylsche Exponentialsummen in der neueren Zahlentheorie, Mathematische Forschungsberichte 15, Berlin (1963).

Address of the Author ${ }^{(1)}$ : Department for Number Theory and ProbaBILITy Theory, University of Ulm, D-89069 Ulm, GERMANY.

Email address : hamaier@mathematik.uni-ulm.de

Address of the author ${ }^{(2)}$ : School of Mathematics, TifR , Homi Bhabha ROAD, Mumbai- 400 005, INDIA.

Email address : sank@math.tifr.res.in 